\title{
Real Space Renormalization Group Methods and Quantum Groups
}

\author{
Miguel A. Martín-Delgado ${ }^{1, *}$ and Germán Sierra ${ }^{2, \dagger}$ \\ ${ }^{1}$ Departamento de Física Teórica I, Universidad Complutense, 28040-Madrid, Spain \\ ${ }^{2}$ Instituto de Matemáticas y Física Fundamental, Consejo Superior de Investigaciones Científicas, \\ Serrano 123, 28006-Madrid, Spain
}

(Received 2 August 1995)

\begin{abstract}
We apply real space renormalization group (RG) methods to study two quantum group invariant Hamiltonians, that of the $X X Z$ model and the Ising model in a transverse field (ITF) defined in an open chain with appropriate boundary terms. The quantum group symmetry is preserved under the RG transformation except for the appearance of a quantum group anomalous term which vanishes in the classical case. We obtain correctly the line of critical $X X Z$ models. In the ITF model the RG flow coincides with the tensor product decomposition of cyclic irreducible representations of $\mathrm{SU}_{q}(2)$ with $q^{4}=1$.

PACS numbers: 75.10.Jm, 05.50.+q, 64.60.Ak
\end{abstract}

Real space renormalization group ( $\mathrm{RG}$ ) methods, as applied to quantum many-body Hamiltonians, originated from the successful study of the Kondo problem by Wilson [1]. Later on people working in field theory and condensed matter generalized it to other problems by using the Kadanoff's concept of block [2,3]. The Block method (BRG) has the advantage of being conceptually and technically simple, but it lacks numerical accuracy or may even produce wrong results. For this reason the analytical BRG methods were largely abandoned in the 1980s in favor of numerical methods such as the quantum Monte Carlo approaches. In the last few years there have been new developments in the numerical RG methods motivated by a better understanding of the errors introduced by the splitting of the lattice into disconnected blocks. A first step was put forward in [4] where a combination of different boundary conditions applied to every block led to the correct energy levels of a simple tight-binding model. This method, however, has not been generalized to models describing interactions. A further step in this direction was undertaken by White in [5] where a density matrix algorithm (DMRG) is developed. The main idea is to take into account the connection of every block with the rest of the system when choosing the states which survive the truncation procedure. The standard prescription is to choose the lowest energy states of the block Hamiltonian. Instead, in the DMRG method one replaces the block Hamiltonian by a block density matrix and chooses the eigenstates of this matrix with the highest eigenvalues. The density matrix is constructed out of the ground state of a superblock which contains the desired block.

In this Letter we propose another RG method which uses the concept of quantum groups. This mathematical notion emerged in the study of integrable systems and it has been applied to conformal field theory, invariants of knots and manifolds, etc. [6,7]. The new application of quantum groups that we envisage has been partially motivated by the aforementioned work of White, Noack, and collaborators [4] and it is probably related to it. This relation is suggested by the fact that quantum groups describe symmetries in the presence of nontrivial boundary conditions. The typical example to understand this property of quantum groups is given by the 1D Heisenberg Ising model with the anisotropic parameter $\Delta$. The isotropic model $\Delta= \pm 1$ is invariant under the rotation group $\mathrm{SU}(2)$, but as long as $|\Delta| \neq 1$ this symmetry is broken down to the rotation group $\mathrm{U}(1)$ around the $z$ axis. One can "restore" this full rotation symmetry by adding appropriate boundary operators to the Hamiltonian of the open chain. The classical group $\mathrm{SU}(2)$ becomes then the quantum group $\mathrm{SU}_{q}(2)$, where the quantum parameter is related to the anisotropy by $\Delta=\left(q+q^{-1}\right) / 2[8,9]$.

These features of $q$ groups made them specially well suited to implement a RG method which takes into account the correlation between neighboring blocks. Let us show how this can be done explicitly in two examples in 1D: the Heisenberg Ising model and the Ising model in a transverse field (ITF).

Heisenberg Ising model ( $X X Z$ model). - The open spin chain Hamiltonian is defined as

$$
\begin{aligned}
& H_{N}(q, J)= \sum_{j=1}^{N-1} h_{j, j+1}(q, J), \\
& h_{j, j+1}(q, J)=\frac{J}{2}\left[\sigma_{j}^{x} \sigma_{j+1}^{x}+\sigma_{j}^{y} \sigma_{j+1}^{y}\right. \\
& \\
& \quad+\frac{q+q^{-1}}{2} \sigma_{j}^{z} \sigma_{j+1}^{z} \\
&\left.\quad-\frac{q-q^{-1}}{2}\left(\sigma_{j}^{z}-\sigma_{j+1}^{z}\right)\right],
\end{aligned}
$$

where $\vec{\sigma}_{j}$ are standard Pauli matrices acting at the $j$ th site of the chain. For the time being $q$ is an arbitrary complex parameter. Observe that the successive terms in $\sigma_{j}^{z}-\sigma_{j+1}^{z}$ in (2) when added into the total $H$ only 
gives boundary operators proportional to $\sigma_{1}^{z}-\sigma_{N}^{z}$ plus the standard bulk Hamiltonian.

Let us now introduce the $q$-group generators of $\mathrm{SU}_{q}(2)$ acting in the spin chain of $N$ sites [9].

$$
\begin{aligned}
& S^{z}=\frac{1}{2} \sum_{j=1}^{N} \sigma_{j}^{z}, \\
& S^{ \pm}=\sum_{j=1}^{N} q^{-1 / 2\left(\sigma_{1}^{z}+\cdots+\sigma_{j-1}^{z}\right)} \sigma_{j}^{ \pm} q^{1 / 2\left(\sigma_{j+1}^{z}+\cdots+\sigma_{N}^{z}\right)},
\end{aligned}
$$

which satisfy the quantum group algebra

$$
\left[S^{+}, S^{-}\right]=\left(q^{2 S^{z}}-q^{-2 S^{z}}\right) /\left(q-q^{-1}\right) .
$$

The important fact is that not only the whole Hamiltonian (1) but also the site-site Hamiltonian (2) commutes with the generators (3) and (4) of $\mathrm{SU}_{q}(2)$ :

$$
\left[h_{j, j+1}, S^{z}\right]=\left[h_{j, j+1}, S^{ \pm}\right]=0 \quad \forall j .
$$

Hence the eigenstates of $H_{N}(q, J)$ can be classified according to the representations of $\mathrm{SU}_{q}$ (2) (see [9] for details).

To construct a real space RG for the Hamiltonian (1) we shall choose blocks of three sites. This is important in order to get a renormalized Hamiltonian of the same form as the original one. The block Hamiltonian involving the first three sites is simply

$$
H_{B}=h_{12}+h_{23} \text {. }
$$

Now we can apply $q$-group representation theory to diagonalize $H_{B}$. There are three energy levels corresponding to the $q$-tensor product decomposition $1 / 2 \otimes 1 / 2 \otimes 1 / 2=$ $1 / 2 \oplus 1 / 2 \oplus 3 / 2$.

For $q$ real and positive (i.e., $\Delta \geq 1$ ) or $q$ a phase (i.e., $|\Delta| \leq 1$ ) the lowest energy level of (7) is doubly degenerate and corresponds to one of the spin $1 / 2$ irreducible representations (irreps), which reads explicitly

$$
\begin{aligned}
\left|\frac{1}{2}\right\rangle= & \frac{1}{\sqrt{2\left(q+q^{-1}+1\right)}} \\
& \times\left(-q^{-1 / 2}|\downarrow \uparrow \uparrow\rangle+\left(q^{1 / 2}+q^{-1 / 2}\right)\right. \\
\left|-\frac{1}{2}\right\rangle= & \frac{1}{\sqrt{2\left(q+q^{-1}+1\right)}} \\
& \quad \times\left(q^{1 / 2}|\downarrow \downarrow \uparrow\rangle-\left(q^{1 / 2}+q^{-1 / 2}\right)\right. \\
& \left.\quad \times|\downarrow \uparrow \downarrow\rangle+q^{1 / 2}|\uparrow \downarrow \downarrow\rangle\right)
\end{aligned}
$$

whose energy is $e_{B}=-(\mathrm{J} / 2)\left(q+q^{-1}+2\right)$.

If we take the $q \rightarrow 0^{+}$limit in (1) and (2) we obtain an Ising model Hamiltonian $(\Delta \rightarrow \infty)$ with a unique ground state given by the Néel state $|\downarrow \uparrow \downarrow \uparrow \ldots\rangle$ [notice that this uniqueness is due to the boundary term $\left(q-q^{-1}\right)\left(\sigma_{1}^{z}-\right.$ $\left.\sigma_{N}^{z}\right) / 2$ ]. On the other hand, for a block of three sites there are four states of lowest energy $(|\uparrow \downarrow \uparrow\rangle,|\downarrow \uparrow \uparrow\rangle,|\downarrow \uparrow \downarrow\rangle$, and $|\downarrow \downarrow \uparrow\rangle)$, while for a block of four sites there is again only one ground state given by $|\downarrow \uparrow \downarrow \uparrow\rangle$. This means that choosing an odd number of blocks is not appropriate to study the Ising limit of (2). To do so one should choose an even number of sites, but this will not be pursued here. Hence we shall concentrate on $q$ being a phase.

The renormalization prescription consists in choosing the states (8) and (9) as the spin up $|\uparrow\rangle^{\prime}$ and down $|\downarrow\rangle^{\prime}$ states associated to the whole block as if it were a single site. Using the standard methods of BRG we obtain the following RG-transformation laws for the spin operators $\vec{S}_{i}$ acting at the sites $i=1$ and 3:

$$
\begin{aligned}
& \left(S_{i}^{x}\right)_{\mathrm{RG}}=\xi(q) S^{\prime x}, \quad i=1,3, \\
& \left(S_{i}^{y}\right)_{\mathrm{RG}}=\xi(q) S^{\prime y}, \quad i=1,3, \\
& \left(S_{i}^{z}\right)_{\mathrm{RG}}=\xi(q) S^{\prime z}+\eta_{i}(q) 1^{\prime}, \quad i=1,3,
\end{aligned}
$$

where $\xi(q)$ is a renormalization factor which depends upon $q$ as

$$
\xi(q)=\left(q+q^{-1}+2\right) / 2\left(q+q^{-1}+1\right)
$$

and

$\eta_{1}=-\eta_{3} \equiv \eta(q)=\left(q-q^{-1}\right) / 4\left(q+q^{-1}+1\right)$.

The multiplicative renormalization factor $\xi(q)$ is common to all the spin operators $\vec{S}_{i}$ as a consequence of the full symmetry group $\mathrm{SU}_{q}(2)$. The "quantized" feature of $\mathrm{SU}_{q}(2)$ is reflected in the "quantum group anomaly" term in (12)-(14), which indeed shows the deviation from the classical case $(q=1)$. Equations (10)-(12) are quite different from the standard BRG analog for the Heisenberg Ising model done in Ref. [10], where the RG equations for $S^{x}$ and $S^{y}$ differ from those of $S^{z}$ (i.e., $\xi^{x}=\xi^{y} \neq \xi^{z}$ ). Using Eqs. (10)-(12) we can get the renormalized block-block Hamiltonian $h_{3 k, 3 k+1}$. Putting all terms together we arrive at the following effective Hamiltonian $H^{\prime}$ which acts on the chain having $N / 3$ sites,

$$
\begin{aligned}
H^{\prime}= & H_{N / 3}\left(q^{\prime}, J^{\prime}\right)+\frac{N}{3} e_{B}(q, J) \\
& +\left(\frac{N}{3}-1\right) e_{B B}(q, J),
\end{aligned}
$$

where

$$
\begin{aligned}
q^{\prime} & =q, \\
J^{\prime} & =\xi^{2}(q) J, \\
e_{B}(q, J) & =-\frac{J}{2}\left(2+q+q^{-1}\right),
\end{aligned}
$$




$$
e_{B B}(q, J)=J \frac{\left(q-q^{-1}\right)^{2}\left(3 q+3 q^{-1}+4\right)}{16\left(q+q^{-1}+1\right)^{2}} .
$$

The $e_{B}$ contribution to the energy comes from the block part $H_{B}$ while $e_{B B}$ is a novel contribution coming from the quantum group anomaly. The remarkable feature of Eqs. (15) and (16) is that the coupling constant $q$, or alternatively $\Delta$, does not flow under the $R G$ transformation, while $J^{(m)}$, which is the value of $J$ after $m$ RG steps, goes to zero in the limit where $m \rightarrow \infty$, which in turn implies that the theory is massless. Hence, Eqs. (16) predicts correctly a line of critical models in the range $|\Delta| \leq 1$. These models are described by conformal field theories (CFT) with central extension $c$ less than 1 . If we write the quantum parameter as $q=e^{i \pi /(\mu+1)}$ then $c=1-6 / \mu(\mu+1)$ [11]. The boundary terms in (1) and (2) are responsible for this fact.

A nontrivial check of the validity of our RG method can be given in the case where $q=e^{i \pi / 3}$ for which $c=0$ (percolation limit). The ground state energy can be computed exactly from the constant terms (15) (assume that $N=3^{m}$ and perform $m$ RG steps), and is given by

$$
E_{0}\left(N, q=e^{i \pi / 3}\right)=-\frac{3}{4} N+\frac{3}{4}
$$

This equation coincides with the exact result obtained in [11] using Bethe ansatz. Since the RG method is variational, the previous result implies that we have actually constructed the exact ground state using the $q \mathrm{RG}$ technique, which therefore becomes exact for this particular case. This is consistent with the fact that in the CFT with $c=0$ there is a unique state, namely, the ground state. What the quantum renormalization group (QRG) method does is to pick up that piece of the ground state which projects into a given block.

It can be shown that a consistent representation theory of quantum groups at root of unity (i.e., $q^{\mu+1}=-1$ ) requires the use of truncated tensor products of $q$-group irreps. In the case of $q=e^{i \pi / 3}$ this truncation implies

$$
(1 / 2 \otimes 1 / 2 \otimes 1 / 2)_{q=e^{i \pi / 3}}=1 / 2,
$$

which is precisely the truncation performed when restricting ourselves to the states (8) and (9). Another interesting example is provided by $q=e^{i \pi / 4}$ which corresponds to the critical Ising model $(c=1 / 2)$. The right-hand side for this $q$ in Eq. (21) contains two spin- $1 / 2$ irreps. According to the QRG method the truncation of the spin-3/2 irrep should be a legitimate operation involving no approximation at all. In Ref. [12] the representation theory of $q$ groups was put in one-to-one correspondence with that of rational conformal field theories (RCFT). There it was observed that the truncation inherent in the construction of the RCFT's has a parallel in the truncation of the representation theory of $q$ groups with $q$ a root of unity. The result we have obtained in this Letter suggests that $q$-group truncations can be carried over a RG analysis of $q$-group invariant chains. In other words, using $q$ groups we can safely truncate states in the block RG method. We may summarize this discussion schematically by

$$
\text { QRG truncation } \leftrightarrow \text { RCFT . }
$$

Ising model in a transverse field. - This simple model has been widely used to test the validity of BRG methods $[2,3]$. The Hamiltonian of an open chain is given by $H=\sum_{j=1}^{N-1} h_{j, j+1}$ where

$$
h_{j, j+1}=-\left(J \sigma_{j}^{x} \sigma_{j+1}^{x}+p \sigma_{j}^{z}+p^{\prime} \sigma_{j+1}^{z}\right) .
$$

The standard choice is $p=p^{\prime}=\Gamma / 2$, in which case (23) has four different eigenvalues. The BRG method with a block with two sites chooses just the two lowest ones. However, if $\left(p, p^{\prime}\right)=(\Gamma, 0)$ [or $\left.(0, \Gamma)\right]$, the Hamiltonian (23) has two doubly degenerate eigenvalues $\pm e_{B}\left(e_{B}=\right.$ $\sqrt{J^{2}+\Gamma^{2}}$ ). This choice is not parity invariant but it implements the self-duality property of the ITF model, yielding the exact value of the critical fixed point of the ITF which appears at $(\Gamma / J)_{c}=1$ [13]. In the following we shall make the choice $\left(p, p^{\prime}\right)=(\Gamma, 0)$. This degeneracy of the spectrum of (23) has a q-group origin. The relevant quantum group is again $\mathrm{SU}_{q}(2)$ with $q^{4}=1$. However, the representations involved are not a $q$ deformation of the spin-1/2 irrep as in the previous example, but rather a new class of irreps which only exist when $q$ is a root of unity. They are called cyclic irreps and neither are highest weight nor lowest weight representations as the more familiar regular irreps. If we call $E, F$, and $K$ the generators of $\mathrm{SU}_{q}(2)$, which correspond essentially to $S^{+}, S^{-}$, and $q^{2 S^{z}}$ in the notation of the previous example, then a cyclic irrep acting at a single site of the chain is given by

$$
E_{j}=a \sigma_{j}^{x}, \quad F_{j}=b \sigma_{j}^{y}, \quad K_{j}=\lambda \sigma_{j}^{z},
$$

where $a=\frac{1}{2} \sqrt{\lambda^{2}-1}, b=-\frac{1}{2} \sqrt{1-\lambda^{-2}}$. The parameter $\lambda$ is the label of the cyclic irrep. Using (24) and the addition rule of $\mathrm{SU}_{q}(2)$ we can get the representation of $E, F$, and $K$ acting on the whole chain:

$$
\begin{aligned}
& E=a \sum_{j=1}^{N} \lambda^{j-1} \sigma_{1}^{z} \cdots \sigma_{j-1}^{z} \sigma_{j}^{x}, \\
& F=b \sum_{j=1}^{N} \lambda^{j-N} \sigma_{j}^{y} \sigma_{j+1}^{z} \cdots \sigma_{N}^{z}, \\
& K=\lambda^{N} \prod_{j=1}^{N} \sigma_{j}^{z} .
\end{aligned}
$$

Now it is a simple exercise to check that these operators commute with (23),

$$
\left[h_{j, j+1}, E\right]=\left[h_{j, j+1}, F\right]=\left[h_{j, j+1}, K\right]=0, \quad \forall j
$$


assuming that we choose

$$
\lambda=\Gamma / J .
$$

The last of the equalities in (28) expresses the wellknown $Z_{2}$ symmetry of the ITF model which allows one to split the spectrum of the Hamiltonian into even and odd subsectors. The other two symmetries are new and explain the degeneracy of the spectrum of $h_{j, j+1}$. By all means the whole Hamiltonian $H=\sum_{j} h_{j, j+1}$ is also invariant under (25)-(27). Notice that $H$ differs from the standard ITF simply in a term at one of the ends of the chain. This is the same mechanism as for the $X X Z$ Hamiltonian: one needs properly chosen operators at the boundary in order to achieve quantum group invariance. Similarly as for the $X X Z$ model the RG analysis of the ITF becomes a problem in representation of quantum groups: Blocking is equivalent to tensoring representations. What is the tensor product of cyclic irreps? Here it is important to realize that all cyclic irreps of $\mathrm{SU}_{q}(2)$ have dimension 2, what distinguishes them is the value of $\lambda$. The tensor product decomposition of two cyclic irreps $\lambda_{1}$ and $\lambda_{2}$ is given by

$$
\left[\lambda_{1}\right] \otimes\left[\lambda_{2}\right]=2\left[\lambda_{1} \lambda_{2}\right],
$$

where the 2 means that $\lambda_{1} \lambda_{2}$ appears twice in the tensor product. If we perform a blocking of two sites we will get two cyclic irreps corresponding to $\lambda^{2}$. Then we expect from $q$-group representation theory that the new effective Hamiltonian $h_{j, j+1}^{\prime}$ will have the same form as (23) but with new renormalized coupling constants $J^{\prime}$ and $\Gamma^{\prime}$ satisfying

$$
\lambda^{\prime}=\Gamma^{\prime} / J^{\prime}=(\Gamma / J)^{2}=\lambda^{2} .
$$

This is indeed the result obtained in [13]. We arrive therefore at the conclusion that the $R G$ flow of the ITF Hamiltonian (23) is equivalent to the tensor product decomposition of cyclic irreps of $\mathrm{SU}_{q}(2)$. This $q$-group interpretation of the RG flow is independent of the size of the blocks: For a $n$-site block the RG flow would be $\lambda \rightarrow \lambda^{n}$. The fixed point $\lambda=1$ of (31) describes the critical regime of the ITF Hamiltonian and it corresponds to a singular point in the manifold of cyclic irreps $[14,15]$. At $\lambda=1$ the operators (25)-(27) are still symmetries of the Hamiltonian ( $a, b$ taking any nonzero value) and they recall the Jordan-Wigner map between Pauli matrices and 1D lattice fermions.

All the Hamiltonians analyzed in this Letter are one dimensional, so the quantum groups are of the type that we know. Despite the fact that the Yang-Baxter equation (the precursor of $q$ groups) has a higher dimensional analog called the Zamolodchikov or tetrahedron equation [16], the corresponding high dimensional analog of quantum groups is not known. This fact represents a barrier to a QRG analysis of Hamiltonians defined in dimensions higher than one.
Another possibility, which is suggested by our results, would be to define quantum groups as those which contain symmetries which are anomalous under RG transformations. This definition is independent of the space dimensionality. The quantum anomalous term in Eq. (12), and an analogous term also present in our QRG treatment of the ITF model, gives a discrete realization of this idea. A continuum analog of this anomaly is given by the FeiginFuchs current, which has an anomalous operator product expansion with the energy-momentum tensor [17]. At this point it may be worth recalling the continuous version of quantum groups in CFT in Ref. [18], which uses the Feigin-Fuchs or free field realization of the latter. Putting all these arguments together, we arrive at the conclusion that quantum groups are indeed defined by symmetries anomalous under RG transformations. This point of view about quantum groups may set up the pathway to new developments in the field.

This work was partly supported by CICYT under Contracts No. AEN93-0776 (M. A. M.-D.) and No. PB92-109 and European Community Grant No. ERBCHRXCT920069 (G. S.).

*Electronic address: mardel@fis.ucm.es

†Electronic address: sierra@cc.csic.es

[1] K. R. Wilson, Rev. Mod. Phys. 47, 773 (1975).

[2] S. D. Drell, M. Weinstein, and S. Yankielowicz, Phys. Rev. D 16, 1769 (1977).

[3] R. Jullien, P. Pfeuty, J. N. Fields, and S. Doniach, Phys. Rev. B 18, 3568 (1978).

[4] S. R. White and R. M. Noack, Phys. Rev. Lett. 68, 3487 (1992).

[5] S. R. White, Phys. Rev. Lett. 69, 2863 (1992); Phys. Rev. B 48, 10345 (1993).

[6] V.G. Drinfeld, in Proceedings of the 1986 International Congress of Mathematics, edited by A. M. Gleason (American Mathematical Society, Berkeley, 1987); M. Jimbo, Lett. Math. Phys. 10, 63 (1985); Lett. Math. Phys. 11, 247 (1986).

[7] C. Gómez, M. Ruiz-Altaba, and G. Sierra, "Quantum Groups in Two-Dimensional Physics" (Cambridge University, Cambridge, England, to be published).

[8] E. K. Sklyanin, J. Phys. A 21, 2375 (1988).

[9] V. Pasquier and H. Saleur, Nucl. Phys. B330, 523 (1990).

[10] J. M. Rabin, Phys. Rev. B 21, 2027 (1980).

[11] F. C. Alcaraz, M. N. Barber, M. T. Batchelor, R. J. Baxter, and G. R. W. Quispel, J. Phys. A 20, 6397 (1987).

[12] L. Alvarez-Gaumé, C. Gómez, and G. Sierra, Phys. Lett. B 220, 142 (1989); Nucl. Phys. B330, 347 (1990).

[13] A. Fernández-Pacheco, Phys. Rev. D 19, 3173 (1979).

[14] D. Arnaudon and A. Chakrabarti, Commun. Math. Phys. 139, 461 (1991).

[15] C. de Concini and V. G. Kac, Prog. Math. 92, 471 (1990).

[16] A. B. Zamolodchikov, Commun. Math. Phys. 79, 489 (1981).

[17] V.S. Dotsenko and V.A. Fateev, Nucl. Phys. B240, 312 (1984); Nucl. Phys. B251, 691 (1985).

[18] C. Gomez and G. Sierra, Phys. Lett. B 240, 149 (1990); Nucl. Phys. B352, 791 (1991). 